\title{
Anesthesia Management of a Child with Duchenne's Muscular Dystrophy Posted for Skin Grafting
}

\author{
Apeksha A Gala ${ }^{1}$, Naina Dalvi ${ }^{2}$
}

\begin{abstract}
Children with neuromuscular diseases may often present for anesthesia either as a part of a diagnostic procedure or surgery relating to their underlying disorder or for incidental surgery. The diagnosis may be clear from either a family history or from the clinical and pathological features. We managed to successfully anesthetize an 8-year-old male child who was a known case of Duchenne's muscular dystrophy and was posted for skin grafting surgery of his right lower limb. We would like to emphasize here the benefits of regional anesthesia, in such cases, (caudal in this case) which to a great extent reduces the requirement of general anesthetics used in the procedure, apart from giving adequate analgesia to the patients.
\end{abstract}

Keywords: Caudal anesthesia, Duchenne's muscular dystrophy, Skin grafting.

Research and Innovation in Anesthesia (2020): 10.5005/jp-journals-10049-0079

\section{INTRODUCTION}

Duchenne muscular dystrophy (DMD) is an x-linked recessive disorder caused by a mutation in the dystrophin gene located on chromosome Xp21. ${ }^{1}$ A typical presentation is proximal muscle weakness. Patients develop respiratory distress and cardiomyopathy in the advanced stage of the disease. The patients have associated difficult airway anatomy in the form of macroglossia and limited mobility of the mandible and cervical spine. These patients are at an increased risk of developing extreme hyperthermia, rhabdomyolysis, and hyperkalemic cardiac arrest when exposed to halogenated inhalational anesthetics and depolarizing muscle relaxants. ${ }^{2,3}$ Here, we discuss the successful anesthetic management of an 8-year-old male child with DMD posted for skin grafting of the lower leg who underwent the procedure under general anesthesia supplemented with caudal anesthesia.

\section{Case Description}

An 8-year-old male child, weighing $16 \mathrm{~kg}$ and a known case of DMD was posted for skin grafting of the right lower leg. He had suffered a right metatarsal fracture, following a fall, which was managed conservatively, by applying a cast over the right leg. However, the child developed an ulcer over his right ankle, which increased progressively and eventually needed skin grafting. The parents gave a history of the child being diagnosed with DMD at the age of 4 years after he had gradual bilateral lower limb weakness with pseudohypertrophy of calf muscles and he walked with a limp. On airway examination, the child had a large tongue and Mallampati grade III. Cardiovascular and respiratory systems were within normal limits. Rest all his investigations including serum creatinine and serum electrolytes were within normal limits. On the day of surgery, a written, informed, and valid consent was taken from his father. A new Jackson-Rees circuit was taken and was flushed with oxygen for 20 minutes. The soda-lime was changed and the vaporizers were demounted from the anesthesia machine. Monitoring included noninvasive blood pressure, electrocardiogram (ECG), neuromuscular monitoring, capnography, temperature, and pulse oximeter. The child was induced with injection fentanyl $30 \mu \mathrm{g}$ followed by propofol
1,2Department of Anaesthesia, HBT Medical College and Dr RN Cooper Municipal General Hospital, Mumbai, Maharashtra, India

Corresponding Author: Apeksha A Gala, Department of Anaesthesia, HBT Medical College and Dr RN Cooper Municipal General Hospital, Mumbai, Maharashtra, India, Phone: +91 9820146886, e-mail: appi. apeksha@gmail.com

How to cite this article: Gala AA, Dalvi N. Anesthesia Management of a Child with Duchenne's Muscular Dystrophy Posted for Skin Grafting Res Inno in Anesth 2020;5(1):13-14.

Source of support: Nil

Conflict of interest: None

$35 \mathrm{mg}$. Atracurium $15 \mathrm{mg}$ was administered, once the train of four (TOF) ratio was 0 , to facilitate endotracheal intubation with a portex uncuffed endotracheal tube no 5 . Considering his nature of the surgery, the child was administered caudal anesthesia with $10 \mathrm{~mL}$ of $0.25 \%$ plain ropivacaine. Intraoperatively, the child was maintained on oxygen + nitrous oxide and propofol infusion. However, the requirement of propofol infusion was considerably low due to the effective caudal anesthesia and stable hemodynamics were maintained intraoperatively. $\mathrm{ETCO}_{2}$ has maintained between 30 and $34 \mathrm{~mm} \mathrm{Hg}$. The child was catheterized intraoperatively to measure the urine output. The total duration of surgery was 1.5 hours. The child was adequately and smoothly reversed and extubated only after confirming complete recovery from muscle paralysis (as shown by the NM monitor) and good respiratory efforts. The child was kept in the intensive care unit for 48 hours and was watched for signs of rhabdomyolysis or hyperkalemia and shifted to ward for further care (Fig. 1).

\section{Discussion}

The main anesthetic concerns in a child with DMD are the possibilities of difficult intubation, prolonged duration of neuromuscular block, a possible need for postoperative ventilation, and occurrence of rhabdomyolysis and cardiac arrhythmias when exposed to halogenated volatile anesthetic agents and depolarizing 


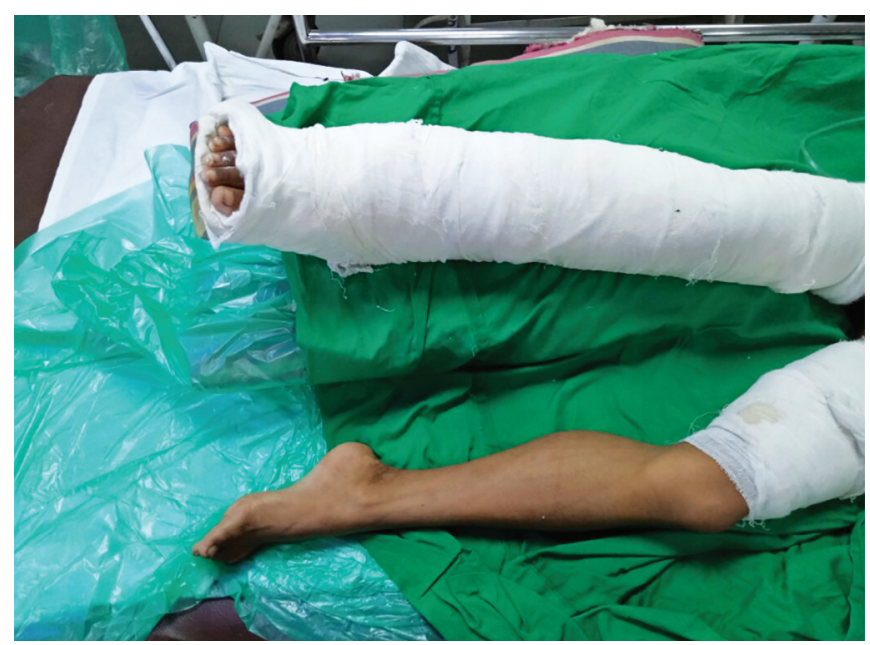

Fig. 1: Pseudohypertrophy of the calf muscles in Duchenne muscular dystrophy

muscle relaxants. Anesthetic considerations in such patients are in relation to the use of various anesthetic agents as well as the concomitant systemic effects of the disease. Such patients usually have multisystemic involvement leading to cardiomyopathy, decreased lung volumes, sleep apnea, pulmonary hypertension, gastric hypomobility and dilation, weak pharyngeal muscles. Use of muscles relaxant in patients with DMD produce effects different than normal patients. The use of succinylcholine is contraindicated due to the possibility of rhabdomyolysis, hyperkalemia, cardiac arrest, and risk of malignant hyperthermia. ${ }^{4}$ Similarly, the use of non-depolarizing neuromuscular drugs is associated with increased sensitivity and prolonged neuromuscular block. The exact cause is still unclear and remains speculative. Two possibilities are considered: (1) change in pharmacokinetics and (2) alteration in neuromuscular junction due to underlying disease. ${ }^{5-7}$ Regional anesthesia is considered a safe option in such cases as it helps not only to provide optimal analgesia but also to minimize the requirements of general anesthetics. ${ }^{8}$ We decided to give general anesthesia along with the use of a non-depolarizing muscle relaxant, considering the presence of macroglossia, and the duration of surgery in our patient. Precautions must be taken to ensure that patient is not exposed to inhalational agents during surgery. This can be ensured by unmounting all the vaporizers from the anesthesia machine, using fresh soda-lime, and breathing circuits by running the machine at a high fresh gas flow rate for 20 to 30 minutes.

\section{Conclusion}

We describe the successful anesthetic management of a case DMD who underwent skin grafting under general anesthesia supplemented with caudal anesthesia. A careful preoperative evaluation and avoidance of anesthetic agents which may trigger rhabdomyolysis and severe hyperkalemia are the keys to successful anesthesia outcomes in patients with DMD.

\section{References}

1. Hoffman EP, Brown Jr RH, Kunkel LM. Dystrophin: the protein product of the Duchenne muscular dystrophy locus. Cell 1987;51(6):919-928. DOI: 10.1016/0092-8674(87)90579-4.

2. Birnkrant DJ, Panitch HB, Benditt JO, et al. American College of Chest Physicians consensus statement on the respiratory and related management of patients with Duchenne muscular dystrophy undergoing anesthesia or sedation. Chest 2007;132(6):1977-1986. DOI: 10.1378/chest.07-0458.

3. Hayes J, Veyckemans F, Bissonnette B. Duchenne muscular dystrophy: An old anesthesia problem revisited. Paediatr Anaesth 2008;18(2):100-106. DOI: 10.1111/j.1460-9592.2007.02302.x.

4. Boltshauser E, Steinmann B, Meyer A, et al. Anaesthesia induced rhabdomyolysis in Duchenne muscular dystrophy. Br J Anaesth 1980;52(5):559. DOI: 10.1093/bja/52.5.559-a.

5. Schmidt J, Muenster T, Wick S, et al. Onset and duration of mivacurium induced neuromuscular block in patient with Duchenne muscular dystrophy. Br J Anaesth 2005;95(6):769-772. DOI: 10.1093/bja/aei249.

6. Blake DJ, Kroger S. The neurobiology of Duchenne muscular dystrophy: learning lesions from muscles. Trends Neurosci 2000;23(3):92-99. DOI: 10.1016/s0166-2236(99)01510-6.

7. Grady RM, Zhou H, Cunningham JM, et al. Maturation and maintenance of neuromuscular synapse:genetic evidence for roles of dystrophin glycoprotein complex. Neuron 2000;25(2):279-293. DOI: 10.1016/s0896-6273(00)80894-6.

8. Aldwinckle RJ, Carr AS. The anaesthetic management of patient with Emery Dreifuss muscular dystrophy for orthopaedic surgery. Can J Anaesth 2002;49(5):467-470. DOI: 10.1007/BF03017922. 